E. CLOUD MOTIONS AND ATMOSPHERIC DYNAMICS 


\title{
ESTIMATES OF BOUNDARY LAYER PARAMETERS IN THE ATMOSPHERES OF THE TERRESTRIAL PLANETS*
}

\author{
G. S. GOLITSYN \\ Institute of Atmospheric Physics, Soviet Academy of Sciences, Moscow, U.S.S.R.
}

\begin{abstract}
The similarity theory of atmospheric boundary layers is applied to an estimate of the form of vertical profiles of average wind velocity and potential temperature in the atmospheres of the terrestrial planets in day- and night-time conditions.

It is then considered, as in the case of the earth, that the magnitude of the turbulent heat flux $q_{T}$ during the day is about 0.1 of $q(1-A)$, where $q$ is the solar constant for the planet and $A$ is its albedo; at night, $q_{T}$ is several times smaller still. The friction velocity $u_{*}$ is taken equal to $2-5 \%$ (depending upon the stratification) of the mean wind velocity in the free atmosphere, which was adopted from previous calculations (Golitsyn, 1968).

The boundary layers in the atmospheres of Mars and Venus and in the hypothetical atmosphere of Mercury are examined in detail. Sharp temperature drops are characteristic of Mars within a few tens of meters from the surface, attaining a magnitude of several tens of degrees, especially during the day. Large changes of the wind velocity also take place in this thin lower layer. This effect results from the low density of the Martian atmosphere.

For Venus, owing to the very high density of the atmosphere, the stratification is close to neutral, i.e., the temperature profile is close to the adiabatic one and the wind profile is of a logarithmic shape.

Owing to high winds, the stratification on Mercury must also be close to neutral with respect to the wind (the profile being close to the logarithmic), but because of the expected low density, the temperature changes near the ground may still be very great.
\end{abstract}

1. The theory of an atmosphere's boundary layer has been sufficiently well worked out (Obukhov, 1946; Monin and Obukhov, 1965; Monin and Yaglom, 1965; Zilitinkevich et al., 1967). A large amount of empirical results has been assembled in terrestrial conditions, particularly in the lower part of the boundary layer, that is, the atmospheric surface layer, or the layer of constant turbulent momentum and heat fluxes, corroborating the conclusions of theory. For the terrestrial atmosphere, the main direction of research is the obtaining of estimates of turbulent fluxes of the momentum $\tau$ and heat $q_{T}$ according to measurements of mean velocity $u(z)$ and temperature $T(z)$ profiles for the atmospheric surface layer. It is also possible to use the data on the geostrophic wind velocity $U_{g}$ and on the potential temperature change $\delta \theta$ for the boundary layer of the atmosphere, in which Coriolis forces already exert a substantial influence. It would be interesting to obtain at least an estimate of the mean temperature and velocity profiles in the boundary layers of other planets.

Do we presently have the data required for such approximate estimates of the structure of the boundary layers on other planets? It seems to us that such data are already available. The mean characteristic velocities of motions in the atmospheres of the planets have already been estimated (Golitsyn, 1968) [see also Golitsyn, 1969]. Consequently, we may evaluate the friction velocity $u^{*}=\sqrt{\tau / \rho}$, where $\rho$ is the density.

* Translated and reprinted from: Izv. Akad. Nauk. S.S.S.R., Fizika Atmosfery i Okeana, Tom 5, No. 8, pp. 775-781, Izdatel'stvo 'NAUKA', 1969. 
Depending upon the stratification (Zilitinkevich et al., 1967), in the terrestrial atmosphere we have $u_{*} / U_{g} \approx 2-5 \%$ (the first value being associated with strong stability, and the second with strong instability, i.e., convection).

A limitation exists on the magnitude of the second parameter determining the structure of the boundary layer, i.e., on the turbulent heat flux: it cannot exceed $q(1-A)=q_{A}$, where $q$ is the solar constant for the planet and $A$ is its albedo. For the earth, even in conditions of strongly developed convection, the ratio $q_{T} / q_{A}$ is of the order of 0.1 . At stable stratification, when the atmosphere is warmer than the ground, which is usually observed at night, $q_{T}<0$, i.e., the heat flux is directed toward the soil and the modulus of the ratio $q_{T} / q_{A}$ is generally considerably smaller than in the daytime. For other planets (Mars, Venus, and perhaps Mercury) there is no reason to expect very substantial departures from the regularities associated with the terrestrial atmosphere. Moreover, one may qualitatively estimate in which direction any such departures could be occurring in these planets.

Therefore, we are confronted by a problem which in a certain sense is the inverse of the problem in the terrestrial atmosphere: having some kind of idea about the magnitude of the momentum and heat fluxes, one must estimate the thickness of the boundary layer and determine the mean vertical profiles of velocity and temperature.

2. According to the general theory (Obukhov, 1946; Monin and Obukhov, 1954; Monin and Yaglom, 1965), the structure of turbulence in a temperature-stratified medium is determined by the following parameters: $q^{\prime}=q_{T} / c_{p} \rho$ is the normalized turbulent heat flux, $u_{*}=\sqrt{\tau / \rho}$ is the friction velocity, and the buoyancy parameter $g \beta$, where $g$ is the gravitational acceleration and $\beta$ is the volumetric expansion coefficient, equal to $1 / T_{0}$ for an ideal gas, where $T_{0}$ is the characteristic temperature of the medium. From these parameters one may construct the scale of length

$$
L=-u_{*}^{3} /\left(\kappa g \beta q_{T} / c_{p} \rho\right)
$$

usually called the 'Monin-Obukhov' scale, and the scale of temperature

$$
T_{*}=q_{T} / c_{q} \rho \kappa u_{*},
$$

where $\kappa$ is the von Karman constant.

The vertical profiles of the mean velocity and potential temperature $\theta=T+\gamma_{a} z$, where $\gamma_{a}$ is the adiabatic temperature gradient, are universal functions of dimensionless height $\zeta=z / L$, whereupon

$$
\begin{aligned}
& u(z)=\kappa^{-1} u_{*}\left[f_{u}(z / L)-f_{u}\left(z_{0} / L\right)\right], \\
& \theta(z)=\theta_{0}+T_{*}\left[f_{0}(z / L)-f_{\theta}\left(z_{0} / L\right)\right],
\end{aligned}
$$

where $z_{0}$ is the roughness parameter. For the universal functions $f_{u}$ and $f_{\theta}$ we have the following expressions (Monin and Obukhov, 1954; Monin and Yaglom, 1965):

$$
f_{u}(\zeta)=f_{\theta}(\zeta)= \begin{cases}\ln \zeta+\beta \zeta, & 0<\zeta, \\ \ln |\zeta|+\beta^{\prime} \zeta_{1}, & \zeta_{1} \leqslant \zeta \leqslant 0, \\ a+C \zeta^{-1 / 3}, & \zeta<\zeta_{1} .\end{cases}
$$


According to careful statistical processing of a vast amount of empirical data (Zilintikevich and Chalikov, 1968 ), $\kappa=0.43 ; \beta=9.9 ; \beta^{\prime}=1.45 ; \zeta_{1}=0.16 ; a=0.24$; $C=1.25$.

These formulas are valid for the atmospheric surface layer, where one may neglect the variation of $\tau$ and $q_{T}$ with height. An estimate is given in Monin and Obukhov (1954) of the thickness $H$ of the atmospheric surface layer under that assumption:

$$
H<\alpha u_{*}^{2}(0) / l U_{g},
$$

where $\alpha=\left[u_{*}^{2}(0)-u_{*}^{2}(H)\right] / u_{*}^{2}(0)$ is the relative variation of friction stress $\tau, l$ is the Coriolis parameter, and $U_{g}$ is the geostrophic wind velocity. For the terrestrial atmosphere, we obtain at $\alpha=20 \%$ and $u_{*} / U_{g} \approx 5 \%, H \approx 50 \mathrm{~m}$. For Mars, with the same value for $\alpha$, we obtain $H \approx 100-200 \mathrm{~m}$, since the Coriolis parameter has the same value, while the mean wind velocities are two to four times higher (Golitsyn, 1968; Golitsyn, 1969). For the slowly-rotating Venus and Mercury we may take for the thickness of the atmospheric surface layer, or, to be more precise, of the boundary layer, the altitude at which the wind velocity is comparable with that in the free atmosphere. Usually, as will be seen below, this thickness is of the order of a few units of the Monin-Obukhov scale $L$.

For the earth and Mars, one may determine the planetary boundary layer inside which the wind velocity varies little in modulus compared with the surface layer, but where, owing to the action of Coriolis forces, a notable wind turn with altitude takes place. The thickness of this layer may be determined as (Zilitinkevich et al., 1967):

$$
L_{*}=\kappa u_{*} / l \text {. }
$$

For the earth, $L_{*}$ is of the order of $1 \mathrm{~km}$, for Mars, it is 2 to 4 times greater. The wind's rotation angle with height depends on the stratification parameter $\mu=L_{*} / L=$ $\kappa^{2} \beta T_{*} / l u_{*}$. In terrestrial conditions (Zilitinkevich et al., 1967), the total angle of wind rotation with height is of the order of several degrees under convective conditions, and attains a value of approximately $40^{\circ}$ under conditions of strong stability (rise of potential temperature with altitude).

A parameter whose value is entirely unknown for other planets enters into formulas (3) and (4), viz., the height of the dynamic roughness $z_{0}$ of the planet's surface. Fortunately, it enters logarithmically, and thus for our purposes an approximate estimate of its magnitude is sufficient. In terrestrial conditions, we have on the average for dry land $z_{0} \approx 1 \mathrm{~cm}$; for oceans, depending upon the sea state, it may be substantially lower; even for a forest $z_{0} \lesssim 1 \mathrm{~m}$. Bearing in mind that for the other planets there are neither oceans nor forests, we shall assume $z_{0} \approx 1 \mathrm{~cm}$.

Being aware of temperature and velocity profiles, we may determine the stability parameter, namely, the Richardson number

$$
R i=g \beta(\mathrm{d} \theta / \mathrm{d} z) /(\mathrm{d} u / \mathrm{d} z)^{2}=\zeta \phi(\zeta),
$$

where the universal function $\theta(\zeta)$ is defined as

$$
\phi(\zeta)=\kappa z u_{*}^{-1} \mathrm{~d} u / \mathrm{d} z=z T_{*}^{-1} \mathrm{~d} \theta / \mathrm{d} z .
$$


At the same time, it is assumed that the coefficients of turbulent exchange for the momentum $K$ and heat $K_{T}$, introduced according to the equalities

$$
\tau=\rho K(\mathrm{~d} u / \mathrm{d} z), q_{T}=-c_{p} \rho K_{T}(\mathrm{~d} \theta / \mathrm{d} z),
$$

are identical. Note that during conditions of strong stability this is specifically not so, and one must then introduce in the denominator of the right-hand part of formula (8) the multiplier $\alpha$, which is the inverse Prandtl turbulence number $\left(\alpha=K_{T} / K\right)$. The universal function for the velocity and temperature will also differ by that multiplier $\left(f_{u}=\alpha f_{\theta}\right.$, see Monin and Yaglom (1965)). In view of the great uncertainty of a series of other factors and the estimative character of the present paper, we shall not take this effect into account here.

The turbulent exchange coefficient or turbulent eddy viscosity $K=K u_{*} L R i$ is expressed by the following formulas:

$$
\begin{array}{ll}
K=\kappa u_{*} z, & |L| \rightarrow \infty, \\
K=\kappa u_{*} z(1+\beta z / L)^{-1}, & |L|<\infty, \\
K=3 C^{-1} u_{*} z(z / L)^{1 / 3}, & \zeta=Z / L<\zeta_{1} .
\end{array}
$$

3. Table I contains the values of solar energy flux $q_{A}$ arriving at each planet's surface: these values are for Mars, Venus, and Mercury, and for the sake of comparison, the earth. The Table also contains the values of the characteristic scale of temperature $T_{*}$ and velocity of atmospheric motions $U$, taken from Golitsyn (1968) or Golitsyn (1969), the normalized turbulent heat flow $q_{T}^{\prime}=q_{T} / c_{p} \rho$, the buoyancy parameter $g / T_{0}$ and the Monin-Obukhov scale $L$. The value of $q_{T} / q_{A}$ was taken equal to 0.1 , which, as was earlier noted, is valid in the case of the terrestrial atmosphere for noontime in conditions of strong convection. During the night, $q_{T}$ and $T_{*}$ will be several times smaller, with another sign, and $L$ will be considerably larger. In the morning and evening the stratification becomes close to neutral, and then $L \rightarrow \infty$, i.e., the boundary layer becomes logarithmic. For Mars we assumed the minimum atmosphere model with a surface pressure $p_{0}=5 \mathrm{mb}$, and for Venus we adopted $p_{0}=100 \mathrm{~atm}$. For the hypothetical atmosphere of Mercury we considered that $p_{0}=1 \mathrm{mb}$. The ratio $u_{*} / U$ was taken equal to $3 \%$.

TABLE I

\begin{tabular}{lcccclc}
\hline Planet & $\begin{array}{l}q_{A} \\
\mathrm{cal} / \mathrm{cm}^{2} \mathrm{~min}\end{array}$ & $\begin{array}{l}U \\
\mathrm{~m} / \mathrm{sec}\end{array}$ & $\begin{array}{l}q_{T}^{\prime} \\
\mathrm{deg} \mathrm{cm} / \mathrm{sec}\end{array}$ & $\begin{array}{l}T_{*} \\
\mathrm{deg}\end{array}$ & $\begin{array}{l}g \beta \\
\mathrm{cm} / \mathrm{sec}^{2} \mathrm{deg}\end{array}$ & $\begin{array}{l}-L \\
\mathrm{~m}\end{array}$ \\
\hline Mars & 0.6 & 40 & 600 & 10 & 2 & 50 \\
Venus & 0.9 & 0.7 & 0.03 & 0.04 & 1.2 & 150 \\
Mercury & 12 & 200 & 10 & 40 & 1 & 600 \\
Earth & 1.2 & 10 & 7 & 1 & 3.3 & 20 \\
\hline
\end{tabular}

The data of Table $\mathbf{l}$ show that the basic parameters determining the structure of the atmospheric surface layer, the friction velocity $u_{*}$, and particularly the scale of 
temperature $T_{*}$ for the planet under consideration differ strongly owing to sharp differences of the fundamental atmospheric parameters, especially the density, so that the atmospheric surface layer on each planet must have its own well expressed features.

Let us now pass to a more detailed examination of these singular features.

\section{A. MARS}

The dynamic and, more particularly, the thermal structure of the lower part of the atmosphere of Mars was considered at fairly great length in Gierasch and Goody (1968). Vertical profiles of temperature and the conditions for convection were computed in detail for various latitudes and seasons, and even for different times of day. One should note that the very same estimate of the mean wind velocity of $40 \mathrm{~m} / \mathrm{sec}$ for a model atmosphere with $p_{0}=5 \mathrm{mb}$ was obtained there by another method than that used in Golitsyn (1968). However, the vertical profiles of the mean wind could not be found in the numerical model considered there, and the authors limited themselves to a very rough estimate of Richardson numbers for various conditions.

In conditions of convection, the 'logarithmic + the linear law' for wind and temperature profiles (5) is valid to values of $\zeta_{1}=-0.16$, i.e., at $L=-50$ to $8 \mathrm{~m}$ altitude from planet's surface. At the same time $\left(z_{0}=1 \mathrm{~cm}\right)$

$$
\begin{aligned}
& u(z)=3[\ln (100 z)-z / 35], \\
& \theta(z) \approx T(z)=T_{0}+10^{\circ}[1(100 z)-z / 35],
\end{aligned}
$$

where $u(z)$ is expressed in $\mathrm{m} / \mathrm{sec}$ and $z$ in $\mathrm{m}$. At $8 \mathrm{~m}$ altitude, $u \approx 20 \mathrm{~m} / \mathrm{sec}$, and $\Delta T=T(0)-T(8 \mathrm{~m}) \approx 60^{\circ}$. At the same time the number $R i \approx-0.03$. Therefore, over an atmospheric layer of the order of $10 \mathrm{~m}$ in all, the velocity attains about one half of the value characteristic of the free atmosphere, while the temperature change reaches $60^{\circ}$ ! (here the difference between the usual temperature $T$ and the potential temperature $\theta$, equal to $\theta=T+\gamma_{a} z$, where for Mars $\gamma_{a} \approx 5 \mathrm{deg} \mathrm{km}^{-1}$ is entirely insignificant). Very sharp, though somewhat smaller temperature variations in daytime and in the lowermost atmospheric layer were also found in Gierasch and Goody (1968). Note that such sharp variations as those found here could not have been obtained in Gierasch and Goody (1968), for in the computation developed there the adopted vertical spacing was $100 \mathrm{~m}$.

Above $8 \mathrm{~m}$ it was necessary to make use of the last formula (5), describing the condition of free convection. At the same time the mean velocity approaches asymptotically its limiting value: the wind velocity in the free atmosphere. According to Monin and Yaglom (1965), this takes place for $\zeta=5$, i.e., $z \approx 250 \mathrm{~m}$. The calculation by the last formula (5) shows that for $\zeta \approx 3$ the velocity reaches about $90 \%$ of its limiting magnitude at infinity. The turbulent exchange coefficient increases rapidly with height. For $z \approx 250 \mathrm{~m}$ we shall have, according to $(12), K \approx 10^{7} \mathrm{~cm}^{2} / \mathrm{sec}$. In Gierasch and Goody (1968), for the lower kilometer layer a value $K \approx 10^{8} \mathrm{~cm}^{2} / \mathrm{sec}$ was obtained. It is apparent that these two estimates are to some degree in accord.

Under conditions of stable stratification (night) we shall assume $L=250 \mathrm{~m}$ and $T_{*}=2^{\circ}$. Then the velocity $40 \mathrm{~m} / \mathrm{sec}$ will be attained at an altitude of about $200 \mathrm{~m}$, and the temperature drop will be $40^{\circ}$. The turbulent exchange coefficient will be of the 
order of $10^{5} \mathrm{~cm}^{2} / \mathrm{sec}$, according to (11). As shown by Gierasch and Goody (1968), at such a value of $K$, the effects of radiative attenuation of temperature can already prove to be substantial. This must lead to a certain decrease of $q_{T}$, i.e., to the increase of $L$, and, by the same token, to a decrease of temperature drop for the given altitude. Note that in Gierasch and Goody (1968), the temperature profiles during the night were determined from purely radiational computations, completely ignoring the turbulence.

In both cases, the velocity above a level of about $200 \mathrm{~m}$ will be nearly constant, but the wind will turn with increasing altitude, approaching the direction of the geostrophic wind at an elevation of $2-4 \mathrm{~km}$. In the daytime, the total rotation angle is small (a few degrees), while at night it may reach several tens of degrees.

\section{B. VENUS}

Owing to great values of $|L|$ one should expect that profiles of potential temperature and velocity should be rather close to logarithmic. Let us estimate at the outset at what altitude the velocity, computed by the formula $u(z)=\kappa^{-1} u_{*} \ln \left(z / z_{0}\right)$ is comparable with the mean velocity of $0.7 \mathrm{~m} / \mathrm{sec}$. This altitude is estimated by the formula $z=z_{0} \exp \left(\kappa u / u_{*}\right)$. Hence, for $z_{0} \approx 1 \mathrm{~cm}$, we have $z \approx 2 \mathrm{~km}$. At the same time, the variation of potential temperature at a distance of the order of $2 \mathrm{~km}$ due to the small value of $T_{*}$ will constitute an entirely insignificant quantity: $\Delta \theta \approx 0.1^{\circ}$, i.e., the temperature profile must be adiabatic to a high degree of precision. At a finite value of $L$, a certain departure from purely logarithmic profiles especially for the velocity, can be observed. It will be manifested mainly in the lowering of the altitude at which the velocity of $0.7 \mathrm{~m} / \mathrm{sec}$ is attained. Thus, under stable conditions (at night), when $q_{T}$ is several times smaller than in the daytime (say 4 times), $L \approx 600 \mathrm{~m}$ and the velocity profile will have the form $u(z)=0.06(\ln 100 z+z / 60)$, where $z$ is expressed in $m$ and $u(z)$ in $\mathrm{m} / \mathrm{sec}$. The velocity $u(z) \approx 0.7 \mathrm{~m} / \mathrm{sec}$ will be attained for $z \approx 100 \mathrm{~m}$. At the same time, $\Delta \theta \approx 0.1^{\circ}$.

For unstable conditions (daytime) $L=-150 \mathrm{~m}$ and the 'logarithmic + linear law' will be observed to $z=-0.16 L \approx 20 \mathrm{~m}$. At this altitude the velocity will reach $0.5 \mathrm{~m} / \mathrm{sec}$. Above this level the velocity profile (and that of potential temperature) will be described by the law $z^{-1 / 3}$, and if one considers that the constant value of velocity is attained for $\zeta=5$, we shall have for the thickness of the layer encompassed by convection $z \approx 750 \mathrm{~m}$. At that distance the variation of potential temperature will be on the order of $0.4^{\circ}$.

Therefore, owing to the great thickness of Venus' atmosphere and the relatively small flux of incident solar radiation, the state of the atmosphere should be close to neutral, i.e., the profile of temperature should be adiabatic and that of velocity logarithmic. The situation will change little especially for the temperature even if we take the limiting, and entirely unrealistic, case $q_{T}=q_{A}$. But, generally speaking, one must bear in mind that if part of the solar radiation is absorbed in the atmosphere of Venus, which is quite probable, then our assertion on the closeness of stratification to a neutral condition will be more correct. But if all the radiation is absorbed in the planet's atmosphere, the boundary layer will be purely logarithmic.

The coefficient of vertical turbulent exchange at an altitude on the order of $1 \mathrm{~km}$ 
will be $K \approx \kappa u_{*} z \approx 10^{5} \mathrm{~cm}^{2} / \mathrm{sec}$. Let us note that for the scale $L=1 \mathrm{~km}$ we could obtain by the Richardson-Obukhov formula a quantity of the same order: $K \approx 0.1 \epsilon^{1 / 3} L^{4 / 3}$, if we take $\epsilon \sim 10^{-3} \mathrm{~cm}^{2} / \mathrm{sec}^{3}$, which is the quantity obtained in Golitsyn (1969) from global estimates of the effectiveness of Venus' atmosphere as a whole in the reprocessing of the solar energy arriving at the planet into mechanical power, i.e., into the generation of kinetic energy of atmospheric motions. Then $K \approx 5 \times 10^{4} \mathrm{~cm}^{2} / \mathrm{sec}$.

\section{MERCURY}

If an atmosphere indeed exists on Mercury, the processes in it will prove to be the most exotic of all the planets of the solar system. The enormous wind velocities, of the order of $200 \mathrm{~m} / \mathrm{sec}$, the differences in temperature between the dark and illuminated sides of the planet of the order of $500^{\circ}$, the length of the day and night corresponding to 180 terrestrial days, all this is instrumental in rendering the boundary layer unique also. Owing to great friction, say $u_{*} \approx 12 \mathrm{~m} / \mathrm{sec}$ (!) the characteristic Monin-Obukhov scale $L$ will be very great also. This is why the velocity profile is found to be close to logarithmic although certain small deflections from the purely logarithmic profile may take place. The velocity reaches a magnitude of the order of $200 \mathrm{~m} / \mathrm{sec}$ over the extent of the lower 100-200 m. At the same time, the high value of $T_{*}$ results in high temperature drops. Thus, in the daytime, the jump of potential temperature, determined by formulas (3)-(5), using the parameters of Table I, yields $\Delta T \approx 400^{\circ}$ over the extent of the lower $100 \mathrm{~m}$ (at a surface temperature of the order of $650^{\circ}$ ); at night, with a surface temperature of approximately $150^{\circ}$, this drop is of the order of $50^{\circ}$ for the lower $200 \mathrm{~m}$. These estimates appear to be extremes, for owing to a very long duration of the day and of the night, the radiation may strongly diminish the temperature drops. In other words, on Mercury, the fraction $q_{T} / q_{A}$ is probably notably less than 0.1 . At the same time, the scale of $L$ will be still greater, i.e., the velocity profile will be still closer to logarithmic, and the boundary layer will be correspondingly thicker.

\section{References}

Gierasch, P. and Goody, R. A.: 1968, Planetary Space Sci. 16, No. 5.

Golitsyn, G. S.: 1968, Izv. AN SSSR, Fizika atmosfery i Okeana 4, No. 11.

Golitsyn, G. S.: 1969, this volume, p. 304.

Monin, A. S. and Obukhov, A. M.: 1954, Trudy Geofiz. In-ta AN SSSR, No. 24.

Monin, A. S. and Yaglom, A. M.: 1965, Statisticheskaya gidromekhanika, part 1, ch. 4, Izd-vo 'Nauka'.

Obukhov, A. M.: 1946, Trudy Inst. teoret. geofiziki AN SSSR, 1.

Zilitinkevich, S. S. and Chalikov, D. V.: 1968, Izv. AN SSSR, Fizika atmosfery i Okeana 4, No. 3.

Zilitinkevich, S. S., Laykhtman, D. L., and Monin, A. S.: 1967, Izv. AN SSSR, Fizika atmosfery $i$ Okeana 3, No. 3. 